\title{
Utilizing Risk Readmission Assessment Tool for Nonhospice Palliative Care Consults in Heart Failure Patients
}

\author{
Tracy Fasolino, PhD, FNP, ACHPN ${ }^{1}$ and Melissa Phillips, MSN, FNP ${ }^{2}$
}

\begin{abstract}
Background: Heart failure (HF) affects 5.1 million U.S. citizens with approximately half dying within 5 years of diagnosis, but many never receive palliative care consults or interventions.

Objective: The purpose of this study was to review medical records of patients admitted and readmitted to the hospital with a primary diagnosis of HF, calculate the risk readmission assessment tool (RRAT) score to determine whether the patient was at high risk, and to determine whether a palliative care consult had been initiated. The tool scoring and medical record review were in retrospect of the hospital admission.

Methods: A retrospective analysis was performed on 70 patients with primary diagnosis of HF who were readmitted at least one time during fiscal year 2012. The RRAT score was calculated on index admission. Patients with high risk scores were assessed for palliative care consult.

Results: Forty-nine of the 70 patients scored high risk for readmission at their index admission. The other 21 patients scored moderate risk at the index admission but subsequently scored high risk on their second admission. A total of 35 patients received palliative care consults. Only 10 of those patients received palliative care consults on the index admission.

Conclusion: Many healthcare providers are unsure when to consult nonhospice palliative care (NHPC) teams for patients with chronic diseases. The findings of this study are important, as they suggest an RRAT can assist with identifying patients with HF in need of NHPC consults.
\end{abstract}

\section{Introduction}

$\mathbf{N}$ EARLY 5 MILLION AMERICANS are currently living with heart failure (HF) with the annual incidence approaching 10 per 1000 individuals over the age of 65 years. ${ }^{1}$ Treating HF costs $\$ 32$ billion annually, primarily because of hospitalization with a significant portion occurring during the last year of life. ${ }^{2} \mathrm{HF}$ is the leading cause of hospitalization among adults over 65 years of age in the United States. ${ }^{3}$ Annually, more than 1 million patients are hospitalized with a primary diagnosis of $\mathrm{HF}$, accounting for a total Medicare expenditure exceeding $\$ 17$ billion. ${ }^{4,5}$ Despite dramatic improvement in outcomes with medical therapy, readmission rates after $\mathrm{HF}$ hospitalization remain high, with more than $50 \%$ readmitted to hospital within 6 months of discharge. ${ }^{6,7}$ Reducing readmissions because of HF has been a primary focus of healthcare systems since the Center for Medicare \& Medicaid Services (CMS) introduced the Hospital Readmission Reduction Program. ${ }^{8}$ Multiple initiatives have been implemented to help reduce readmissions because of $\mathrm{HF}$, including incorporating a risk readmission assessment tool (RRAT), partnering with community physicians, closer communication with the patient, and integration of palliative care services. ${ }^{9,10}$

The role of a nonhospice palliative care (NHPC) team is improving quality of life and assisting patients and family members with serious, complex chronic illnesses, such as HF. The NHPC team uses a multidisciplinary approach to addresses patients' and caregivers' physical, emotional, and spiritual needs. HF is notorious for variable prognosis and heavy symptom burden, so these patients should be assured access to NHPC teams. Nevertheless, many patients with HF

\footnotetext{
${ }^{1}$ School of Nursing, Clemson University, Clemson, South Carolina.

${ }^{2}$ Upstate Cardiology, Bon Secours Medical Group, Greenville, South Carolina.

Accepted April 13, 2016.
}

(C) Tracy Fasolino and Melissa Phillips 2016; Published by Mary Ann Liebert, Inc. This Open Access article is distributed under the terms of the Creative Commons Attribution Noncommercial License (http://creativecommons.org/licenses/by-nc/4.0/) which permits any noncommercial use, distribution, and reproduction in any medium, provided the original author(s) and the source are credited. 
do not receive palliative care consults. The current workforce of palliative care providers, while growing, is challenged to meet the growing demand of patients with serious illnesses. ${ }^{10}$

At the time of this study, hospital providers consulted palliative care solely on clinical judgment. In particular, the cardiology team was unsure when to request palliative care involvement. In an effort to guide palliative care consults, the cardiology and palliative care teams evaluated the RRAT as a possible resource to trigger palliative consults. The RRAT had been implemented with specific disease populations within the health system as a way to identify patients at greatest risk for readmission. The tool had been used effectively as a method for resource allocation. The teams decided to use the RRAT to help guide palliative care consults but requested completion of a retrospective study to determine how many patients scoring high risk had received palliative care consult during their hospitalization. The purpose of this study was to review medical records of patients admitted and readmitted to the hospital with a primary diagnosis of HF, calculate the RRAT score, and determine whether a palliative care consult had been initiated. The tool scoring and medical record review were in retrospect of the hospital admission.

\section{RRAT}

Before this study, the RRAT had been developed from a statistical prediction model using variables thought to contribute to early readmission. ${ }^{11}$ The model found that the top predictors for readmission were insurance status, marital status, having a regular physician, number of admissions within the last calendar year, current length of stay, the score on Charlson comorbidity index score, and the physical Short Form-12. ${ }^{9}$ Each predictor had an associated beta coefficient that was transformed to a point system (beta coefficient $\times 10$, rounded to nearest integer). The health system used the top six elements from the prediction model and determined high, moderate, and low risk for readmission during a separate research project (Appendix A. Risk Readmission Assessment Tool).

\section{Methods}

This study investigated the usefulness of the RRAT as a guide for NHPC consults for patients diagnosed with HF who scored high risk for readmission. If the patient scored in the high risk category, the research team extracted the medical record to see whether a palliative care consult had been ordered. If so, the research team reviewed outcomes of the consult, with particular attention to end-of-life wishes, including establishing do not resuscitate (DNR) and hospice enrollment. The facility's institutional review board approved the study.

\section{Results}

A total of 565 patients with a primary diagnosis of HF were admitted during fiscal year 2012. The mean age was 72.5 years with $53 \%$ male. More than $75 \%$ of the patients admitted were Caucasian. Of the 565 patients admitted, 70 were readmitted to the hospital at least once with some patients readmitted up to five times during the fiscal year. A total of 49 patients $(n=70)$ scored high risk on the RRAT with their index admission (81.4\%). Twenty of the remaining patients scored moderate risk on index admission but then subsequently scored high risk on the following admission.

\section{NHPC consult and outcomes}

A total of $35(50 \%)$ palliative care consults were placed on the 70 patients scoring high risk for readmission. Of the 35 patients receiving palliative care, only $10(14 \%)$ of these patients received consults on their index admission and only $3(4 \%)$ received subsequent palliative care consults on their second admission. The remaining 22 patients $(66 \%)$ received palliative care consults during the third, fourth, or fifth admission. Of those patients receiving palliative care consults $(n=35), 15$ patients $(21 \%)$ enrolled in either home hospice or a hospice facility. These patients were identified by the NHPC as eligible for hospice enrollment. The number of NHPC visits completed before establishment of the goal for hospice enrollment ranged from one to five visits. An average of three contacts by the NHPC medical team (1 contact $=1$ day consult or rounding by the MD or NP) was needed to establish the goal of hospice. The team assisted with establishing end-of-life choices, particularly DNR with seven patients and up to three contacts with the patient were needed to establish the goal.

\section{Discussion}

Many healthcare providers are unsure when to consult NHPC teams for patients with chronic diseases. The findings of this study are important, as they suggest an RRAT can assist with identifying high risk patients with $\mathrm{HF}$ in need of NHPC consults. Using the RRAT as a guide for care management allows healthcare systems to target available resources for those patients at greatest need. NHPC teams can access these patients and family members during hospitalization to build relationships and trust. The goals of care and end-of-life wishes for these patients may not be determined at initial contact by the NHPC team but can be established after subsequent encounters.

As this study revealed, numerous encounters by the NHPC team may be necessary with the patient and family members to establish goals. Discussions regarding goals of care and end of life can be very difficult and require repeated encounters by the NHPC. Many healthcare providers may not appreciate the complexity of the services provided by NHPC teams and associated time required to educate, establish goals, and outline the decisions related to end of life.

Many patients with HF and their caregivers have minimal understanding of this chronic condition and the trajectory of the disease. In addition, these patients deal with a tremendous symptom burden and poor quality of life. Ideally, NPHC should have early encounters with patients diagnosed with chronic illnesses, such as the index admissions, subsequent admissions, and outpatient settings. However, the number of palliative care providers is sparse, so primary care providers with training in palliative care would be ideal to manage this patient population.

Due to the notoriously variable prognosis of HF, enrollment into hospice services continues to be sparse. This study found a moderate enrollment into hospice. Patients and family members may not be aware of the role of hospice for nonmalignant diseases attributing to the lack of knowledge of available services. Once introduced to hospice services for nonmalignant disease, the patients and family members were accepting of hospice enrollment. In addition, many of these patients were eligible for hospice but had not been introduced 
to the services by their cardiologist and/or primary care provider. The initial discussion of hospice services occurred during hospitalization by the NHPC team. Lastly, the use of NHPC teams for management of symptoms associated with chronic illnesses continues to evolve. Many healthcare providers are unaware that NHPC teams are trained for assisting with symptom burden and use various interventions, aside from opiate prescribing.

One limitation of this study is the retrospective nature of evaluating the RRAT as a method for identifying patients at high risk for readmission and determining whether palliative care consults are appropriate. In reviewing patients with high risk scores, consults for palliative care were found for only half of these patients. Conceivably, the other patients who did not receive consults but scored high risk could have benefited for services by the palliative care team. The role of NHPC team in symptom management for the high risk patients with HF was not clearly identified in the medical records. While establishing end-of-life choices and assisting with hospice enrollment, these patients experience a heavy symptom burden.

\section{Conclusion}

There has been an increase in prevalence of HF in the U.S. population over the past 10 years. Identifying patients with $\mathrm{HF}$ at high risk for readmissions allows healthcare systems to mobilize resources quickly and efficiently. NHPC teams play a key role in addressing the symptom burden associated with $\mathrm{HF}$, as well as addressing end-of-life wishes and hospice services. A RRAT can assist healthcare providers to identify those patients with the greatest need for NHPC consults and care management.

\section{Author Disclosure Statement}

No competing financial interests exist.

\section{References}

1. Mozaffarian D, Benjamin EJ, Go AS, et al.: Heart disease and stroke statistics-2015 update: A report from the American Heart Association. Circulation 2015;131:e29-e322.

2. Heart Failure Fact Sheet: Centers for Disease Control and Prevention (2015). Retrieved January 13, 2015. www.cdc .gov/dhdsp/data_statistics/fact_sheets/fs_heart_failure.htm (last accessed January 13, 2015).
3. Center for Medicare \& Medicaid Services: Readmissions Reduction Program. CMS.gov 2014 (last accessed February 1, 2015).

4. Rosamond W, Flegal K, Furie K, Greenlund, K, et al.: Heart disease and stroke statistics-2008 update: A report from the American Heart Association Statistics Community and Strok Statistics Subcommittee. Circulation 2008; 117:e25-e146.

5. Krumholz HM, Merrill AR, Schone EM, Schreiner GC, et al.: Patterns of hospital performance in acute myocardial infarction and heart failure 30-day mortality and readmission. Cir Cardiovasc Qual Outcomes 2009;2:407-413.

6. Joynt KE, Jha AK: Who has higher readmission rates for heart failure, and why? Implications for efforts to improve care using financial incentives. Circ Cardiovasc Qual Outcomes 2011;4:53-59.

7. Chun S, Tu JV, Wijeysundera HC, Austin PC, et al.: Lifetime analysis of hospitalizations and survival of patients newly-admitted with heart failure. Circ Heart Fail 2012. DOI: 10.1161/CIRCHEARTFAILURE.111.964791. http://circheartfailure.ahajournals.org

8. U.S. Department of Health and Human Services: Better, Smarter, Healthier: In historic announcement, HHS sets clear goals and timeline for shifting Medicare reimbursements from volume to value. Press release, January 26, 2015. www.hhs .gov/news/press/2015pres/01/20150126a.html (last accessed January 26, 2015).

9. Bradley B, Curry L, Horwitz L, et al: Hospital strategies associated with 30-day readmission rates for patients with heart failure. Circ Cardiovasc Qual Outcomes 2016;9:445-450.

10. America's Care of Serious Illness. 2015 State-by-state report card on access to palliative care in our Nation's hospitals. Center for advancement of palliative care. Retrieved December 1, 2015. https://reportcard.capc.org/ (last accessed December 1, 2015).

11. Hasan O, Meltzer D, Shimon A, Shaykevlch M, et al.: Hospital readmission in general medicine patients: A prediction model. J Gen Intern Med 2012;25:211-219.

Address correspondence to: Tracy Fasolino, PhD, FNP, ACHPN School of Nursing Clemson University Clemson, SC 29676

E-mail: tfasoli@clemson.edu 
Appendix A. Risk ReAdmission Assessment Tool

Variable

Point allocation

Points

Either Married, Living with SO, Assisted Living, LTAC, SNF or Rehab

Consistent Relationship with Primary Physician Group

2

(Seen at least one time within the past 6 months)

Insurance Source - Include all that apply

Medicare

Medicaid

Self-Pay

Admissions in last calendar year

1 time

2 times

3 times

4 times

5 or greater times

Current length of stay $>5$ days

Charlson Comorbidity Index (CCI): Scoring based on age \& disease

Category 1

- MI (History, Not ECG changes only)

- $\mathrm{CHF}$

- CVA: Mild/No residual/TIA

- Dementia

- Chronic pulmonary disease

- Connective tissue disease

- Peptic Ulcer Disease

- Mild liver disease (without portal HTN, including chronic hepatitis)

- Diabetes without end-organ damage (excludes diet-controlled alone)

Category 2

- Hemiplegia

- Moderate or severe renal disease

- Diabetes with end-organ damage (retinopathy, neuropathy, nephropathy, or brittle diabetes)

- Leukemia (acute or chronic)

- Lymphoma

Category 3

- Moderate or severe liver disease

Category 4

- Metastatic solid tumor

- AIDS (not just HIV positive)

\section{Age Based Category Scoring}

Ages 40-49: 1, 2, 3, 6

Ages 50-59: 2, 3, 4, 7

Ages 60-69: 3, 4, 5, 8 Ages 70-79: 4, 5, 6, 9

Ages 80-89: 5, 6, 7, 10

Ages 90-99: 6, 7, 8, 11 